\title{
Ostwald Ripening: An Approach with Dynamical Systems
}

\author{
F.S. Lameiras \\ Centro de Desenvolvimento da Tecnologia Nuclear - CDTN / CNEN, \\ C.P. 941, 30.123-970 Belo Horizonte, MG - Brazil
}

Received: August 15, 1998; Revised: March 30, 1999

\begin{abstract}
This approach assumes three functions independently acting on a set of microparticles. The first one, $w_{1}$, concerns re-distribution of mass to decrease the surface energy. The second one, $w_{2}$, concerns re-distribution of mass to increase the entropy of the microparticle set. The third one, w3, is a further re-distribution of mass that vanishes a microparticle. Once vanished, its mass is distributed among its neighbors. $w_{1}$ and $w_{3}$ release energy, whereas $w_{2}$ absorbs energy. Part of the energy released should be available to sustain $w_{2}$. The action frequency of $w_{1}, w_{2}$, and $w_{3}$, the amount of mass exchanged in each iteraction, the fraction of released energy available to sustain $w_{2}$, and the size of a vanishing microparticle can be varied. As the dynamical system formed by $w_{1}, w_{2}$, and $w 3$ act on an initial microparticle set, it is observed an evolution resembling the Ostwald ripening concerning steady-state size distribution and microparticle growth.
\end{abstract}

Keywords: Ostwald ripening, dynamical systems, simulation

\section{Introduction}

The decrease surface energy is usually assumed as the driving force for the Ostwald ripening, so that when two microparticles interact with each other by exchanging mass, the larger one grows at the expense of the smaller one. To handle the whole set of microparticles, it is generally assumed that the microparticles are in an average environment, and that there is a critical size, $r_{c}$, so that a microparticle larger than $r_{c}$ will always grow, and one smaller than $r_{c}$ will always shrink ${ }^{1}$. Although it is recognized that this approach is only "statistically true", and that there are local interactions not following this rule, these mean field approaches neglect these events. Moreover, the collective behavior should also be taken into account through the entropy of the microparticle set. So, in addition to the decrease of the surface energy, its distribution among the microparticles should be considered. To maximize the entropy of the set, this distribution should be uniform, and the microparticles should also be uniformly distributed in space. This is the configuration for the microparticle set with the highest probability if one assumes an intrinsic random re-distribution of mass between the microparti$\mathrm{cles}^{2}$. Consequently, there is also an intrinsic tendency in the interactions between microparticles to achieve all of them with the same form and size. This tendency should compete with the one to decrease the surface energy ${ }^{2,3,4}$.
A microparticle releases mass at a rate depending on its solubility, which is given by the Gibbs-Thomson relation, but it also absorbs mass released by other microparticles at a rate depending on its surface area, the concentration of released mass at its position, and the reactions involved in the absorption process. The energy necessary to increase the surface area as consequence of the absorption is available from the energy released when the surface area of another microparticle is decreased due to the release of mass. The mass release and absorption are treated here separately and as independent of each other.

Some computer simulations are based on interactions between two microparticles. In these approaches the larger microparticle grows at the expense of the smaller one, so that a microparticle can grow when it interacts with one of its neighbors (if it is the larger one), or shrink when it interacts with other neighbor (if it is the smaller one) . $^{5}$. These approaches are an improvement in relation to the mean field theory. But the entropy of the microparticle set is also not taken into account. By considering the interactions in this way, there is no tendency for the distribution of the surface energy among the microparticles to become uniform, because the larger microparticles will always grow at the expense of the smaller ones.

The model proposed here assumes that there are three functions acting upon a set of microparticles independently of one another. The first one, $w_{l}$, concerns re-distribution 
Table 1. The three functions acting upon the set of microparticles.

\begin{tabular}{|c|c|c|}
\hline$w_{1}$ & $w_{2}$ & $w_{3}$ \\
\hline if $v_{1} \geq v_{2}$ then & if $\mathrm{v}_{1} \leq \mathrm{v}_{2}$ then & if $\mathrm{v}_{\mathrm{j}}$ has "vanishing \\
\hline $\mathrm{v}_{1}=\mathrm{v}_{1}+\Delta \mathrm{v}$ & $\mathrm{v}_{1}=\mathrm{v}_{1}+\Delta \mathrm{v}$ & size" then \\
\hline $\begin{array}{l}\mathrm{v}_{2}=\mathrm{v}_{2}-\Delta \mathrm{v} \\
\text { else }\end{array}$ & $\begin{array}{c}\mathrm{v}_{2}= \\
\text { else }\end{array}$ & $\begin{array}{l}\text { for } i=0 \text { to } n \text { do } \\
\qquad v_{i}=v_{i}+v_{j} / n\end{array}$ \\
\hline $\mathrm{v}_{1}=\mathrm{v}_{1}-\Delta \mathrm{v}$ & $\mathrm{v}_{1}=\mathrm{v}_{1}-\Delta \mathrm{v}$ & $\mathrm{v}_{\mathrm{j}}=0$ \\
\hline $\mathrm{v}_{2}=\mathrm{v}_{2}+\Delta \mathrm{v}$ & $\mathrm{v}_{2}=\mathrm{v}_{2}+\Delta \mathrm{v}$ & $\begin{array}{c}\text { else } \\
\text { do nothing }\end{array}$ \\
\hline \multicolumn{2}{|c|}{$\begin{array}{l}\text { where } v_{i}(i=1,2) \text { is the microparticle } \\
\text { volume and } \Delta v \text { is the amount of volume } \\
\text { transferred. }\end{array}$} & $\begin{array}{l}\text { where the } v_{i} \text { 's are } \\
\text { neighbors and } \mathrm{n} \\
\text { their number. }\end{array}$ \\
\hline
\end{tabular}

of mass between two microparticles to decrease the surface energy. This is achieved by taking an amount of volume of the smaller microparticle and adding it to the larger one. The second function, $w_{2}$, also concerns re-distribution of mass between two microparticles, but to increase the entropy of the microparticle set. This is achieved by taking an amount of volume of the larger microparticle and adding it to the smaller one. The third function, $w_{3}$, is a further re-distribution of mass between microparticles, but at this time one microparticle is vanished. This interaction can only happen if the microparticle has a "vanishing size". Once a microparticle is vanished, its mass is distributed among its neighbors. This interaction decreases the number of microparticles and also the surface energy. Table 1 shows these functions. Conservation of volume (or mass) is assured in $w_{1}, w_{2}$, and $w_{3} . w_{1}$ and $w_{3}$ are interactions that release energy, whereas $w_{2}$ absorbs energy. Part of the energy released by $w_{1}$ and $w_{3}$ should be available to sustain $w_{2}$, whereas the other part is released to the environment. Before each action of $w_{2}$ the stock of released energy that is available should be observed. If there is not enough energy, $w_{2}$ cannot act. After each action of $w_{2}$, this stock is diminished by the amount of energy absorbed. When $w_{l}$, $w_{2}$, and $w_{3}$ act simultaneously on a set of microparticles they form a dynamical system ${ }^{6}$. The characteristics of the evolution of this system resembles the Ostwald ripening. It is not the purpose of this paper to make an in-depth analysis based on the theory of dynamical systems. The properties of the dynamical system formed by $w_{1}, w_{2}$, and $w_{3}$ are only intuitively developed.

\section{Implementation}

The microparticles are assumed as distributed in a regular three dimensional lattice, as shown in Fig. 1. The microparticle sizes, measured through a linear dimension, were assumed as smaller than the distance between the lattice nodes, so that a microparticle never touches its neighbors. No other assumption concerning the volume fraction of the microparticles is made. A void lattice node means that it is occupied by a microparticle with volume equal to zero. It was taken a lattice with $21 \times 21$ x $21=9261$

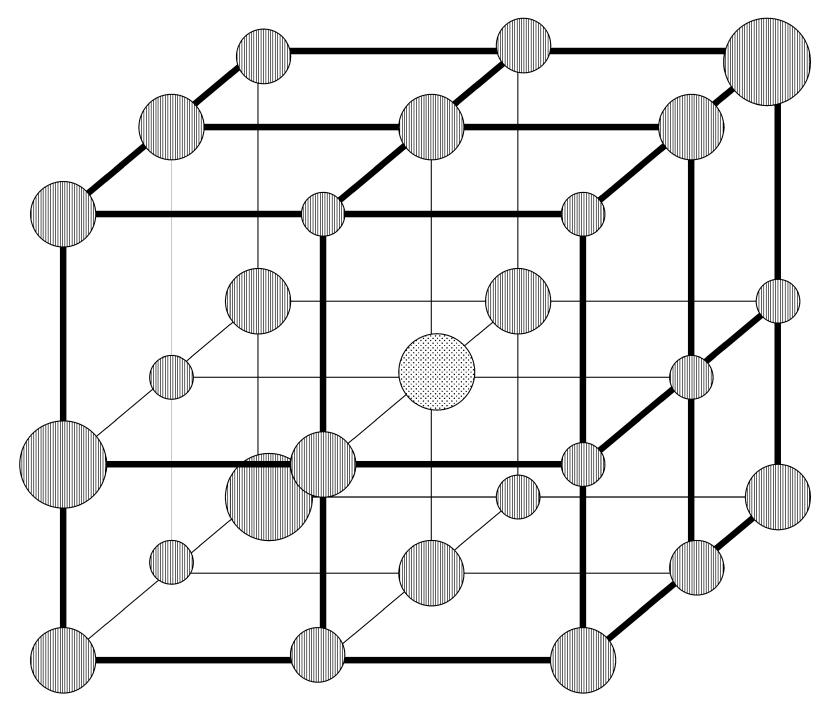

Figure 1. Spatial distribution of the microparticle set assumed in this model.

nodes. An initial set of microparticles with different sizes is distributed among the nodes.

The model works as follows: a) choose at random one of the functions $w_{1}, w_{2}$ or $w_{3} ; b$ ) choose at random one node and apply the function chosen in a) to the microparticle located at it. Case the node is empty, do nothing and proceed to the next iteration. In each iteration, the chosen microparticle interacts with all its 26 neighbors. To check the model, some calculations were performed. $\Delta \mathrm{v}$ was taken as

$$
\Delta \mathrm{v}=0.01\left(1-\frac{\mathrm{v}_{\mathrm{i}}}{\mathrm{v}_{\text {total }}}\right) \mathrm{v}_{\mathrm{i}}
$$

where $v_{\text {total }}$ is the volume of the microparticles altogether and $\mathrm{V}_{\mathrm{i}}$ is the volume of the microparticle that is loosing volume. This equation has no physical meaning and is used only to test the model performance.

\section{Results}

Figure 2 shows the result of the action of $w_{l}$ on an initial microparticle set with the size distribution shown. The diameter is calculated by taking the microparticles as spheres. The mean diameter is calculated from the mean microparticle volume, defined as (volume of all microparticles) / (number of microparticles). If $n$ is the number of microparticles, the final distribution should ideally be one with $n-1$ microparticles with vanishing size and one microparticle with volume close to $v_{t o t}$. This distribution can be seen as a fixed point of $w_{1}$ in the space of size distributions of $n$ microparticles with total volume $v_{t o t}$. Nevertheless, due do the restrictions of the model that allow interactions only with the first neighbors and the spatial correlations developed during the iterations, the final dis- 


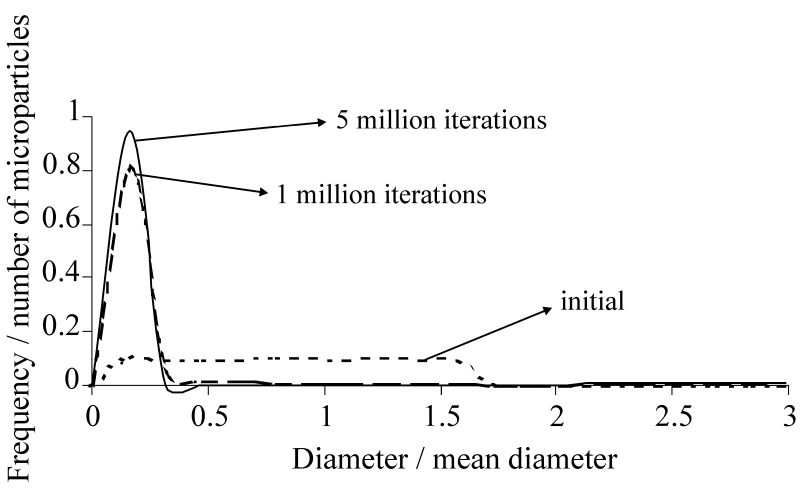

Figure 2. The result of action of $\mathrm{w}_{1}$.

tribution occurs after 5 millions iterations, with a small population at larger sizes (not shown).

$w_{2}$ is not allowed to act alone, because it have to be powered by the energy released by $w_{1}$ or $w_{3}$. There is one exception to this rule in this implementation for the case where both interacting microparticles have the same volume, but the calculations have shown that this has a negligible effect on the resulted size distributions. If enough energy is given, $w_{2}$ also has a fixed point in the space of size distributions above described. It is the distribution with all microparticles with the same size equal to $v_{t o t} t n$.

Figure 3 shows cases where both $w_{1}$ and $w_{2}$ act. Although it is possible to vary the probabilities of action of $w_{1}$ and $w_{2}$, it was considered only the case where these probabilities are equal, because the system doesn't "prefer" any of these functions. According to the theory of dynamical systems, $w_{1}$ and $w_{2}$ form an iterated function system (IFS) that converges to a size distribution for the microparticle set (called attractor), which can be obtained by increasing the number of iterations. This figure suggests that this is the case for the dynamical system formed by $w_{l}$ and $w_{2}$, and that the final size distribution is very similar to the one resulted from the action of $w_{1}$. The effect of $w_{2}$ is to slow down the action of $w_{l}$ (the IFS needs a greater number of iterations to achieve a size distribution). The increase of the fraction of released surface energy available to power $w_{2}$ enhances this effect. There should be a situation where the action of $w_{1}$ could be virtually stopped by $w_{2}$.

Concerning the action of $w_{3}$, the vanishing size, defined as the critical size for the collapse of a microparticle, was arbitrarily established as any size with diameter smaller than $16 \%$ of the initial mean diameter. It can only vanish the smaller microparticles until they are exhausted, what was already observed for 0.5 millions iterations. The IFS formed by $w_{2}$ and $w_{3}$ shows a very similar result. $w_{2}$ can only act while $w_{3}$ delivers energy released by the vanishing of the smaller microparticles. Once they are exhausted, both $w_{2}$ and $w_{3}$ are no more allowed to act.

Figure 4 shows the case of action of both $w_{1}$ and $w_{3}$. The attractor of this IFS was achieved for 12 millions iterations.

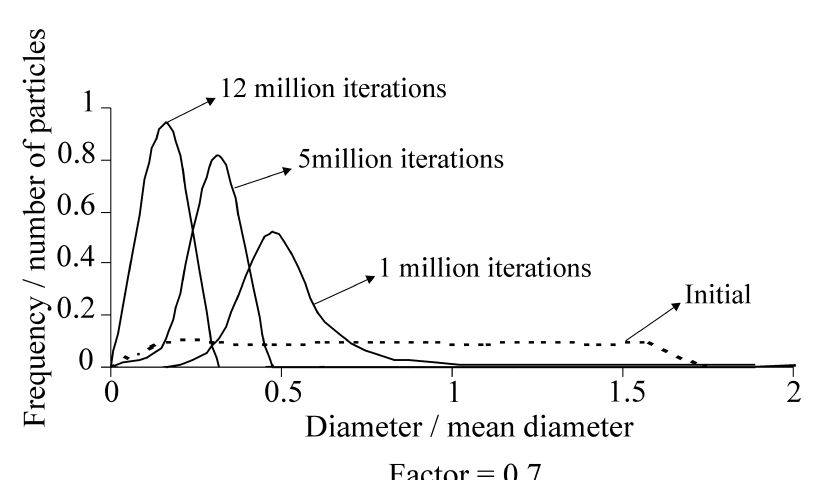

Factor $=0.7$

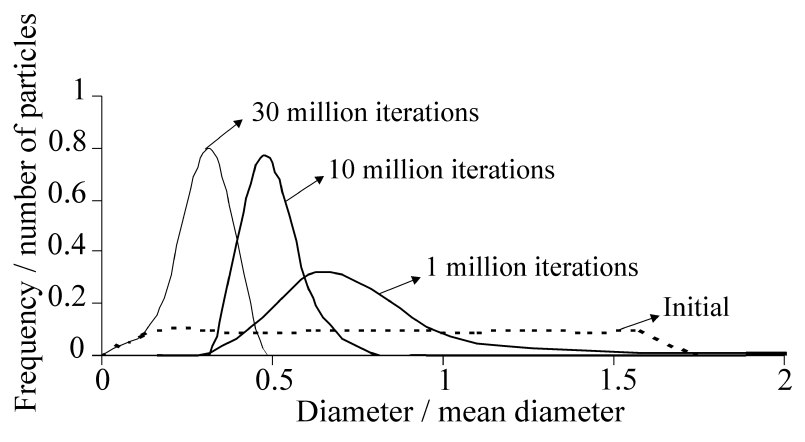

Factor $=0.9$

Figure 3. The action of $\mathrm{w}_{1}$ and $\mathrm{w}_{2}$ for two fractions (factor) of the released surface energy available to power $\mathrm{w}_{2}$.

This figure also shows the increase of the mean volume of the microparticles as a function of the number of iterations. Initially, it was observed a linear growth law. At about 4.5 millions iterations this behavior was disturbed probably due to spatial correlations developed because of the implementation of the model. The growth stopped at 12 millions iterations, when the attractor was achieved.

Figure 5 shows cases for the IFS formed by $w_{1}, w_{2}$, and $w_{3}$. The fraction of released energy available to power $w_{2}$ was considered as in Fig. 3. The attractor was not achieved in these cases because the required number of iterations is prohibitively large. The linear growth law was observed for the mean volume after an "incubation" period. Both the linear growth law and the incubation period are strongly influenced by the fraction of released energy available to power $w_{2}$. Compared to Fig. 4, the effect of $w_{2}$ is to slow down the action of the IFS formed by $w_{1}$ and $w_{3}$ and to decrease the scattering of the size distributions. If $\Delta v$ is doubled, the evolution of the size distribution becomes more rapid, the increase of the mean volume is also more rapid, and the incubation period decreases. The growth stopped at 45 millions iterations.

\section{Discussion}

In this implementation, the number of iterations can be associated with time according to a linear relationship. 

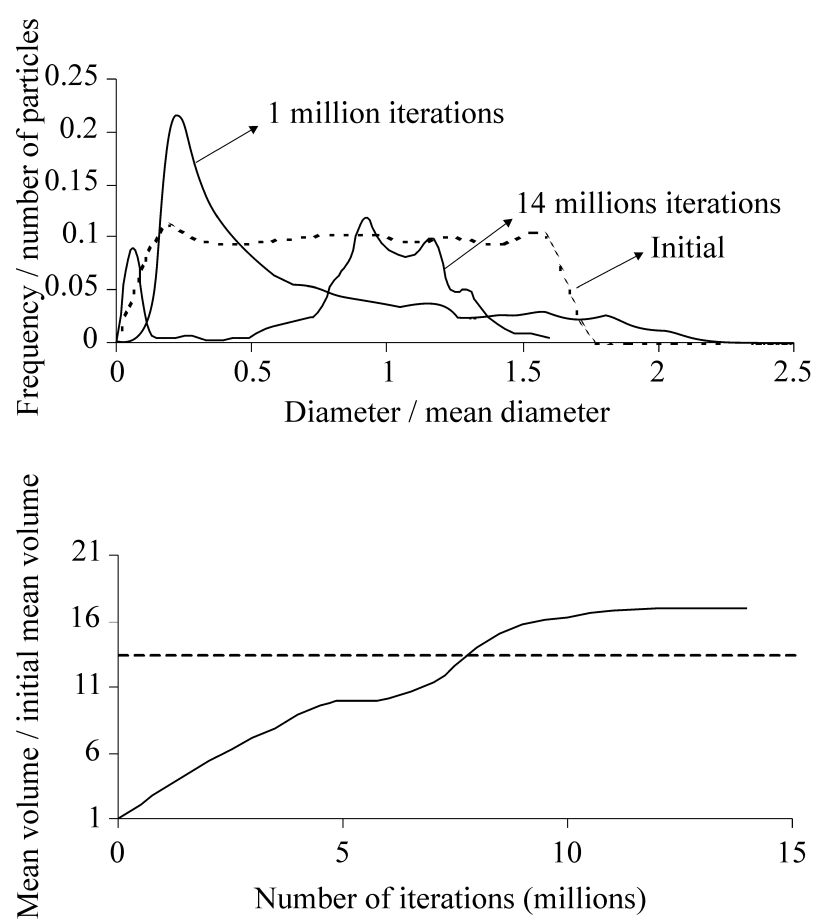

Figure 4. The result of action of $\mathrm{w}_{1}$ and $\mathrm{w}_{2}$.

The fraction of released surface energy available to power $w_{2}$ should depend on the size and geometry of the sample, as well as on the material properties and temperature. Because of its strong influence on the evolution, it is advisable to avoid a simple fit of this fraction to experimental data. It should be derived based on physical backgrounds.

The amount of mass transferred in the interactions between microparticles and the vanishing size should also be appropriately modeled. Different models can be introduced in this implementation.

The growth of the microparticle mean volume shows three features: a) an incubation period, that depends on the fraction of released surface energy available to power $w_{2}$ and on the amount of mass transferred in each interaction between two microparticles. It may even not be observed in some situations, as in Fig. 4; b) a linear growth law after the incubation period, which can be described by

$$
\frac{1}{N}=\left(\frac{1}{N_{0}}\right)\left[1+\alpha\left(t-t_{0}\right)\right]
$$

where $N$ is the number of microparticles at instant $t$; $t$, and $N_{0}$ are the initial instant and number of microparticles, respectively, and $\alpha$ is a constant. This is similar to the grain growth law proposed by Rhines and Craig (7) for the steady-state. Because of this similarity the growth is called "steady-state" when Eq. (2) is observed; c) a perturbation on the growth law of Eq. (2) is observed for larger mean sizes, followed by the full stop of the growth. These events
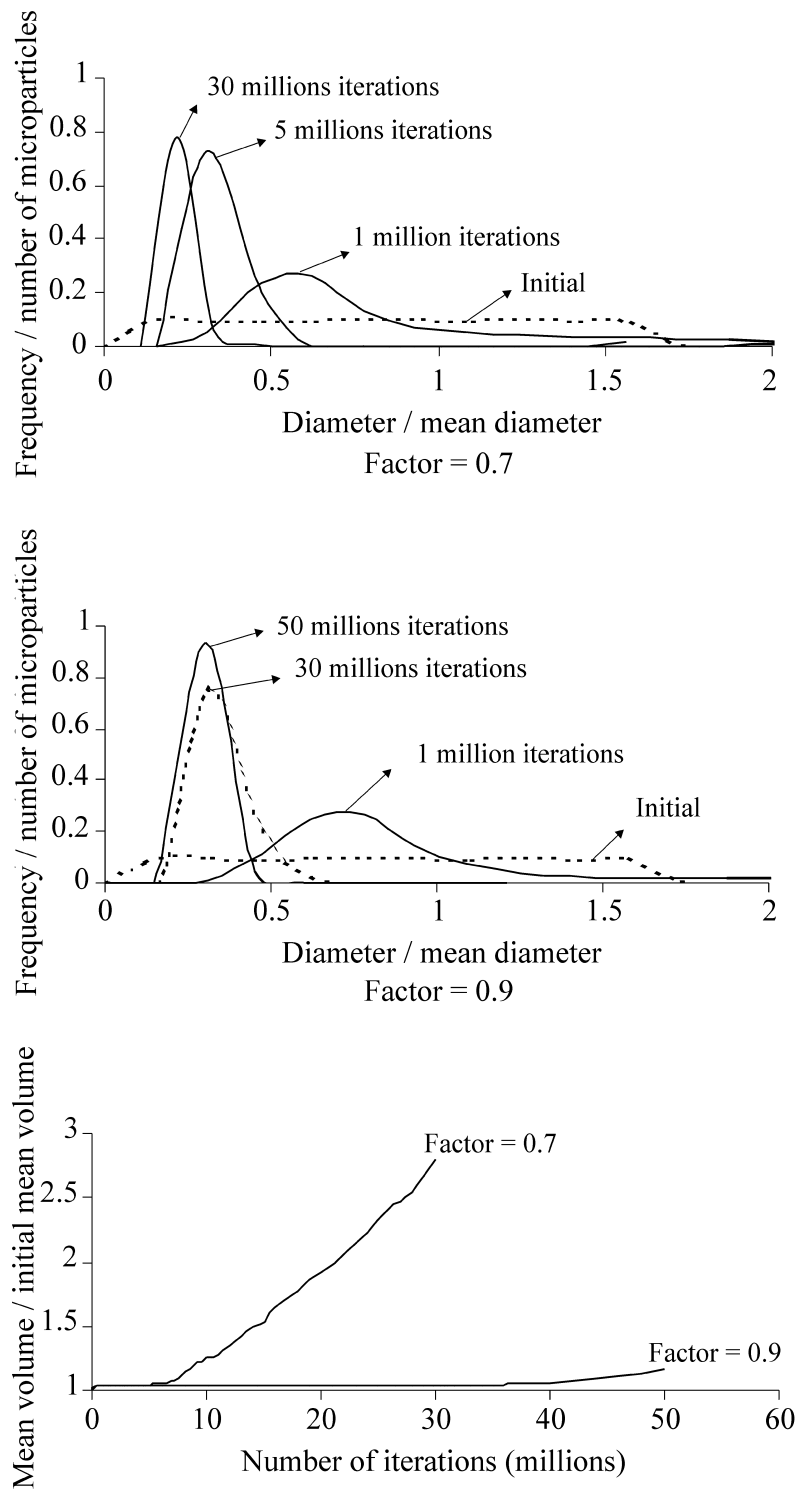

Figure 5. The result of action $\mathrm{w}_{1}, \mathrm{w}_{2}$ and $\mathrm{w}_{3}$.

are related to spatial correlations developed because of this implementation.

After the onset of the linear growth law (steady-state), the size distributions developed for the IFS formed by $w_{1}$ and $w_{2}$, and $w_{1}, w_{2}$, and $w_{3}$ can be described by

$$
n_{i}=n_{0} e^{\left[\lambda\left(d_{0}-d_{i}\right) d_{i}^{2}\right]}
$$

where $n_{i}$ is the number of microparticles in the $i$-th size class with size $d_{i}$, and $n_{0}$ is the number of vanishing microparticles. $\lambda$ and $d_{0}$ are parameters related to the scattering and position of the distribution in the $d_{i}$-axis, respectively. This distribution is derived elsewhere (2). A small population of microparticles at larger sizes was developed. Although this population has slowly decreased as the iterations proceeded, it persisted up to the full stop 
of growth. It cannot be described by Eq. (3). I also explain this population as consequence of the spatial correlations. The steady-state size distributions for the IFS formed by $w_{1}$ and $w_{3}$ cannot be described by Eq. (3). These are more close to the lognormal distribution (see Fig. 4, 1 million iterations).

This implementation works while the microparticles don't touch each other. The volume fractions admitted by this restriction depends on the size and position of the larger microparticles: a) if they are adjacent, the maximum volume fraction admitted can be calculated as maximum volume fraction $=(6 / \pi) * v_{\text {total }} /$ (largest microparticle diameter $)^{3} *$ (number of lattice nodes). For the case of Fig. 4 , this volume fraction is $1.2 \%$; b) if they are not adjacent, larger volumetric fractions can be admitted. It is possible to implement a monitoring routine to check if "collisions" between microparticles take place. While these collisions don't happen, larger volume fractions can be admitted.

\section{Summary}

The iterated function system formed by three functions acting independently of each other on a set of microparticles of different sizes randomly distributed into a three dimensional lattice shows characteristics that resemble the Ostwald ripening. These functions concern the re-distribution of mass during the interactions between microparticles. The first one, $w_{1}$, promotes interactions to decrease the surface energy of the microparticle set. The second one, $w_{2}$, promotes interactions to increase the entropy of the microparticle set. The third one, $w_{3}$, eliminates microparticles of vanishing sizes and distributes their mass among neighbors. $w_{1}$ and $w_{2}$ are interactions that release energy, whereas $w_{2}$ absorbs energy. Part of the energy released by $w_{1}$ and $w_{3}$ should be available to sustain $w_{2}$.

This system was implemented by arbitrarily defining the amount of mass transferred in each interaction, the fraction of released energy available to sustain $w_{2}$, and the vanishing size. Different models can be introduced in place of these arbitrary definitions. The Rhines and Craig's growth law for steady-state was observed after an incubation period with no growth. At later stages this linear growth law is disturbed followed by a full stop of the growth, which are events associated with spatial correlations developed during the iterations. After the onset of the linear growth, the size distributions can be described by Eq. (3) for the iterated function systems formed by $w_{1}$ and $w_{2}$, and $w_{1}, w_{2}$, and $w_{3}$. For the system formed by $w_{1}$ and $w_{3}$ the distributions are more close to the lognormal one.

\section{References}

1. Vengrenovitch, R.D. Acta Metall., v. 30, p. 10791086, 1982.

2. Lameiras, F.S. Scripta Metall., v. 28, p. 1435-1440, 1993.

3. Rivier, N.; Lissowski, A. J. Phys. A: Math. Gen., n. 15, p. L143-L148, 1982.

4. Rivier, N. Phil. Mag. B, n. 52, p. 795, 1985.

5. Hunderi, O.; Ryum, N. Acta Metall., v. 29, p. $1737-$ 1745,1981

6. Barnsley, M. Fractals Everywhere, Academic Press, Inc., San Diego, Ca-USA, 1988.

7. Rhines, F.N.; K.R. Craig. Metallurgical Trans., v. 5, p. 413-425, 1974. 